\title{
On the Modeling and Evaluation of the Physical Layer of HSPA Uplink
}

\author{
Elvis M. G. Stancanelli, Jean M. S. Maciel, Cibelly A. de Araújo, \\ Carlos H. M. de Lima, Emanuel B. Rodrigues, Francisco R. P. Cavalcanti
}

\begin{abstract}
A single-user link-level High-Speed Packet Access Uplink (HSPA UL) static simulation tool is described in this paper. All transport channel processing and spreading/modulation steps are implemented together with Additive White Gaussian Noise (AWGN) radio channel. The focus is on the simulation of the data channel, although the effects of the control channel are taken into account. The Block Error Rate (BLER) is provided by the simulator. Furthermore, an Average Value Interface (AVI)-like Link-to-System (L2S) interface is suggested. The $\mathrm{L} 2 \mathrm{~S}$ interface framework is improved with the introduction of Interference Reduction Factor (IRF).
\end{abstract}

Keywords-HSPA UL, physical layer, simulation tool, performance evaluation.

\section{INTRODUCTION}

The 3rd. Generation Partnership Project (3GPP) Release 6 (R6) specifications of the Wideband Code Division Multiple Access (WCDMA) system include a new uplink transport channel, the Enhanced Dedicated Channel (E-DCH) [1], which provide higher data rates, increased capacity and reduced delays than the Dedicated Channel (DCH). E-DCH supports new features such as short Transmission Time Interval (TTI) (2 ms), fast Hybrid Automatic Repeat Request (HARQ) and fast scheduling [2], [3].

In order to study intelligent solutions for the use of available radio resources in WCDMA Enhanced Uplink systems - also known as High-Speed Packet Access Uplink (HSPA UL) -, performance measures of lower layers must be taken into account. Such measures can be obtained from exhaustive link-level simulation campaigns. So, we developed a flexible and efficient single-user Link-Level High-Speed Packet Access Uplink (LL HSPA UL) static simulation tool using $\mathrm{C}++$ Object-Oriented Programming (OOP) language. Essentially, the LL HSPA UL simulator evaluates the radio link performance of the E-DCH traffic channel, by means of, for instance, Bit Error Rate (BER), Raw Bit Error Rate (RBER), Frame Erasure Rate (FER) or Block Error Rate (BLER) calculation. Temporal structure (slots, frames, and sub-frames), modulation schemes, channel coding strategies, etc., are being modeled in accordance with the 3GPP standards.

Besides of the evaluation of link-level functionalities, the LL HSPA UL simulator can supply the system-level simulator

The authors are with Wireless Telecommunication Research Group GTEL / Federal University of Ceará - UFC, Fortaleza, Brazil, \{emiguel, jean, cibelly, carlos, emanuel, rodrigo\}@gtel.ufc.br.

This work was supported by a grant from Ericsson of Brazil - research branch under ERBB/UFC.17 technical cooperation contract. with Look-up Tables (LuTs). Basically, Link-to-System (L2S) interface solutions aim to map the link-level performance into required metrics on system-level. In this sense, we proposed a modified version of the Average Value Interface (AVI) L2S interface, with the introduction of the Interference Reduction Factor (IRF) concept.

This paper is divided into the following sections: section II introduces the E-DCH modeling, with its channel structures and coding/multiplexing/spreading/modulation chain. Section III describes the link-level simulation tool developed for HSPA UL studies. In section IV we propose and describe the AVI strategy based on the IRF concept. Section V presents an example of link-level performance evaluation of High-Speed Packet Access Uplink. Section VI presents main observations, final conclusions and perspectives.

\section{E-DCH MODEL}

In this section, we present the modeling used for the new HSPA UL transport channel. The characteristics of the transport and physical channels of 3GPP R6 are compared with the channels of Release 99 (R99) in section II-A. General aspects about the transport channel processing of E-DCH are presented in section II-B, while some physical layer processing details about spreading and modulation are given in section II-C.

\section{A. Structure of HSPA UL Channels}

HSPA UL has a new uplink transport channel named E-DCH that supports enhanced features compared to the uplink transport channel of R99 (DCH). In HSPA UL both E-DCH and DCH can coexist in the same User Equipment (UE) and their processing is similar, with two main differences. While there can be only one E-DCH transport channel in the UE that is mapped on a Coded Composite Transport Channel (CCTrCH) of E-DCH type, there may be multiple parallel $\mathrm{DCH}$ that are multiplexed together to a single $\mathrm{CCTrCH}$ of $\mathrm{DCH}$ type. The other difference is the presence of the new HARQ functionality in the E-DCH transport channel processing chain [4]. Although the HSPA UL allows the coexistence of E-DCH and DCH in the same mobile terminal, in this work only the E-DCH chain was modeled.

During the transport channel processing, the E-DCH is mapped to one or multiple parallel new dedicated physical data channels, which are known as E-DCH Dedicated Physical Data Channels (E-DPDCHs). The information bits transmitted in the E-DPDCH are a result of the E-DCH transport 
channel processing, which will be described in more details in section II-B. E-DPDCH has to coexist with all the uplink dedicated channels of the 3GPP Release 5 (R5), which are the Uplink High-Speed Dedicated Physical Control Channel (HS-DPCCH), the Dedicated Physical Data Channel (DPDCH) and the Dedicated Physical Control Channel (DPCCH).

The E-DPDCH has a structure very similar to the R99 DPDCH. They both use Binary Phase-Shift Keying (BPSK) modulation, they can transmit multiple channels in parallel, they follow the same fast power control loop and adjust the number of channel bits to the amount of data actually being transmitted.

The main differences regarding the physical data channels are the spreading factor and the TTI length. E-DPDCH supports a Spreading Factor (SF) of 2, while the minimum SF supported by the DPDCH is 4. The E-DPDCH supports simultaneous transmission of two SF2 and two SF4 codes which both lead to the maximum physical layer bit rate of 5.76 Mbps. Nevertheless, the most pronounced physical layer difference between E-DPDCH and DPDCH is the new TTI length of $2 \mathrm{~ms}$ supported by the former. The $10 \mathrm{~ms}$ radio frame structure already familiar to the DPDCH is maintained, but it is divided into five independent sub-frames when a $2 \mathrm{~ms}$ TTI is used. No matter what is the TTI length, only one E-DCH transport block is transmitted in each TTI [4]. Fig. 1(a) depicts the E-DPDCH frame structure.

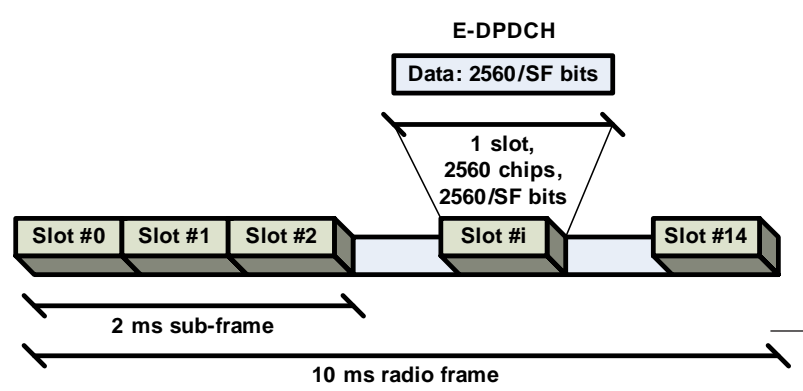

(a) E-DPDCH

E-DPCCH

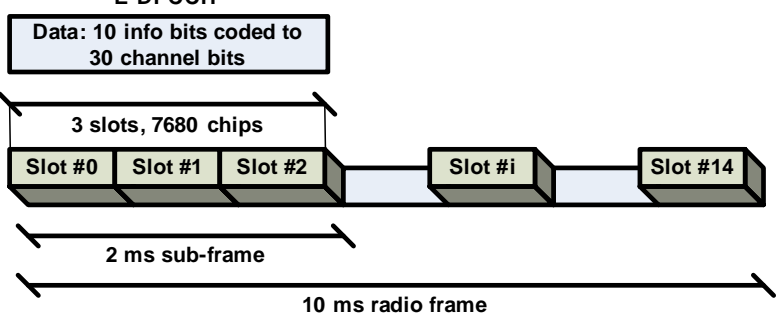

(b) $\mathrm{E}-\mathrm{DPCCH}$

Fig. 1. HSPA UL frame structure

The E-DPDCH is not a standalone channel since it requires simultaneous transmission of two control channels: Dedicated Physical Control Channel (DPCCH) and E-DCH Dedicated Physical Control Channel (E-DPCCH). The former delivers the power control bit needed for downlink power control, as well as transmits pilot bits that are needed for Signal-to-Interference Ratio and channel estimations in the receiver. The latter transmits all the necessary information about the E-DPDCH that is needed in order to know how to receive the data channel. To some extent the E-DPCCH does the same for the E-DPDCH transmission as the DPCCH does for the DPDCH transmission.

The E-DPCCH uses a fixed SF of 256 in order to deliver 30 channel bits in a $2 \mathrm{~ms}$ sub-frame. In each E-DPDCH TTI transmitted, 10 bits of information must be delivered, as indicated in Fig. 1(b). If the TTI length is $10 \mathrm{~ms}$, then the 30-bit E-DPCCH sub-frame is repeated five times in order to reduce the power level [4].

\section{B. Transport Channel Processing}

The technical modeling of the LL HSPA UL simulator is in accordance with the transport channel processing for E-DCH [1] and the uplink spreading/modulation chain [5]. This composition can be seen in the diagram shown in Fig. 2.

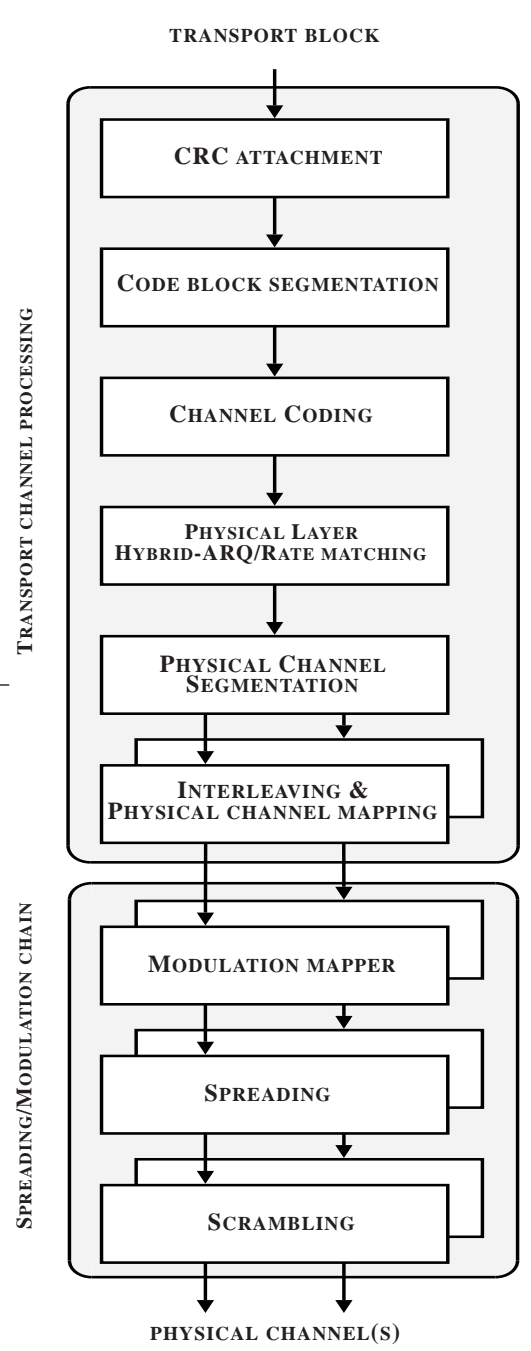

Fig. 2. Transport channel processing and spreading/modulation chain for E-DCH.

At the beginning of the transmission chain, a Cyclic Redundancy Check (CRC) of 24 bits is attached to the transport block, which is checked at the reception chain so that the occurrence of errors is verified for each detected block. 
In a real system, if an error is detected by checking the CRC code parity bits, the retransmission mechanism may act. In this version of the LL HSPA UL simulator, we do not implement such kind of retransmission.

The correction of errors is performed through a turbo encoder with rate $1 / 3$. The turbo encoder uses two parallel convolutional codes with an interleaving device between them. The corresponding channel decoder performs the soft decoding by means of, for instance, Maximum A Posteriori (MAP), log-MAP or log-max algorithms.

A specific Code Block Segmentation module is necessary to be placed between the CRC Attachment and Channel Coding modules in order to adjust the size of the exchanged block. The allowable block size can be from 40 to 5114 bits.

By using either puncturing or repetition of bits, the Hybrid Automatic Repeat Request (HARQ)/Rate Matching (RM) module aims to match the transport channel instantaneous bit rate to the physical channel bit rate. Basically, two functions are performed [1], [6]:

- To match the number of coded bits to the total number of available bits in the E-DPDCH in accordance with the selected E-DCH transport format;

- To generate different sets of encoded bits for the retransmission scheme of the HARQ.

This procedure is essential for achieving the retransmissions at Layer 1 with soft combining, which in HSPA UL is referred as HARQ. Incremental Redundancy (IR) and Chase Combining (CC) are the two possible HARQ modes. As early explained, we have not implemented any retransmission mechanism.

Throughout the chain, bits are interleaved, blocks are segmented and physical channels are mapped. The interleaving of HSPA UL is performed by a block interleaver and consists of bits input to a matrix with padding, the inter-column permutation of the matrix and bits output from the matrix with pruning. Subsequently, the data modulation functionalities (i.e., modulation mapping, spreading and scrambling) are performed to make possible the communication through the radio channel. Their description is presented in the following.

\section{Spreading/modulation chain}

The modulation is simplified as a bit-to-symbol mapper assuring the equivalent base-band assumption. The BPSK modulation is used as a power-efficient modulation scheme [7]. On the other side, in the demodulator, a soft demodulation is performed in order to convert the signal at symbol format to a signal at a bit format preserving a kind of certainty measure.

In the sequel, the spreading module takes place. Spread spectrum intends to increase the robustness of the signal against interference or jamming and to prevent hostile detection. This module is divided into three stages (see Fig. 3): a spreading stage, a weighting stage and an In-Phase and Quadrature (IQ) stage. In the former stage, each E-DPDCH is multiplied by a specific spreading code. Moreover, it is generated and introduced a random symbol sequence that represents the E-DPCCH and, herein, this sequence is multiplied by a specific spreading code with spreading factor
256 [8]. The above mentioned codes are generated by the Orthogonal Variable Spreading Factor (OVSF) code tree [5]. By the moment of the reverse processing at the reception chain, the E-DPCCH is disregarded since it does not transmit data information bits.

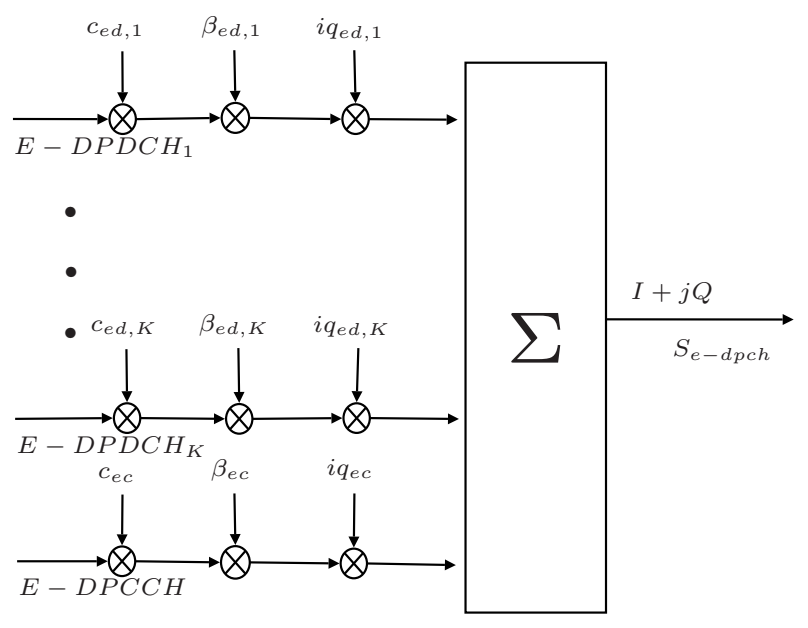

Fig. 3. HSPA UL spreading structure.

In the following stage, E-DPCCH is weighted by an associated $\beta_{e c}$ gain factor. The $k^{t h}$ E-DPDCH is weighted by an associated $\beta_{e d, k}$ gain factor, which is dependent on the E-DCH Transport Format Combination (E-TFC) and whose calculation is described as follows.

Considering that the user is using the $j^{\text {th }}$ E-TFC, we first must find the reference E-TFC related to the $j^{\text {th }}$ E-TFC. Suppose that there are $M$ signaled reference E-TFCs and that $\mathrm{E}-\mathrm{TFCI}_{r e f, m}$ denote the E-DCH Transport Format Combination Indicator (E-TFCI) of the $m^{t h}$ reference E-TFC. Let $\mathrm{E}-\mathrm{TFCI}_{j}$ denote the E-TFCI of the $j^{t h}$ E-TFC. For the $j^{\text {th }} \mathrm{E}$-TFC, we have:

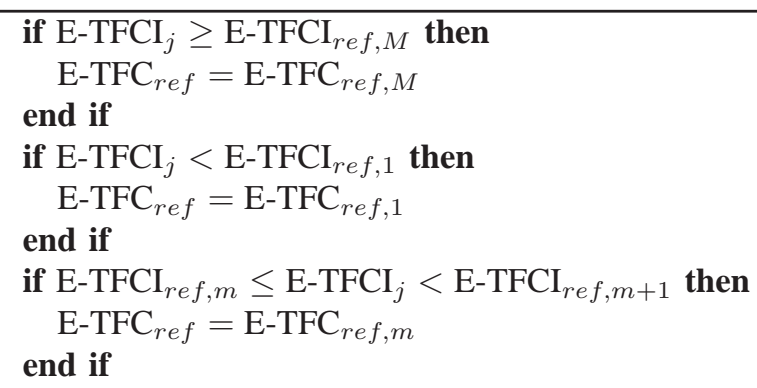

In this way, we can find the reference E-TFC related to the $j^{\text {th }}$ E-TFC, and its corresponding reference gain factor $\beta_{\text {ed,ref }}$. The E-DPDCH gain factor, $\beta_{e d}$, may take a different value for each HARQ offset $\Delta_{\text {harq }}$ [5]. Thus, the temporary variable $\beta_{e d, j, h a r q}$ for the $j^{\text {th }}$ E-TFC is computed as

$$
\beta_{e d, j, h a r q}=\beta_{e d, r e f} \sqrt{\frac{L_{e, r e f}}{L_{e, j}}} \sqrt{\frac{K_{e, j}}{K_{e, r e f}}} 10^{\frac{\Delta_{h a r q}}{20}},
$$

where $L_{e, r e f}$ and $L_{e, j}$ are the number of E-DPDCHs used for the reference E-TFC and the $j^{t h}$ E-TFC, respectively; $K_{e, r e f}$ and $K_{e, j}$ denote the Transport Block Size (TBS) of the reference E-TFC and the $j^{\text {th }}$ E-TFC, respectively; 
The unquantized gain factor $\beta_{e d, k, j, u q}$ for the the $k^{\text {th }}$ E-DPDCH and the $j^{\text {th }}$ E-TFC depends on the spreading factor of the $k^{t h}$ E-DPDCH and uses the $\beta_{e d, j, h a r q}$ calculated from (1) in the following manner:

$$
\begin{aligned}
& \text { if Spreading Factor for E-DPDCH }{ }_{k}==2 \text { then } \\
& \quad \beta_{e d, k, j, u q}=\sqrt{2} \beta_{e d, j, h a r q} \\
& \text { else } \\
& \quad \beta_{e d, k, j, u q}=\beta_{e d, j, h a r q} \\
& \text { end if }
\end{aligned}
$$

The final step is the quantization described in [8], which is dependent on the gain factor $\beta_{c}$ of the DPCCH channel. As a result, we have the $\beta_{e d, k}$ depicted in Fig. 3 .

In the IQ Mapping stage, E-DPDCHs with real-valued signals are mapped to the branch in-phase or quadrature [5]. E-DPCCH with real-valued signals is always mapped to the branch in-phase [5].

At last, the scrambling module acts. Each UE has its own scrambling code, which is generated from a Gold Sequence. Both short and long sequences are allowed. It is in charge of the higher layers to assign the selection of these codes [5].

\section{Simulation TOOL}

The LL HSPA UL simulator was developed using the Link-level Software Development Framework (LSDF) described in [9]. LSDF is a software framework fully implemented in $\mathrm{C}++$ language and using commonly known OOP design pratices. By using LSDF, the implementation effort and codification time are significantly reduced due to the inherent modularity and reusability of this framework, decreasing costs and avoiding reimplementation of either functionalities or entities. The goal of LSDF is to provide a robust and automated framework for software development upon which any link-level simulation can be easily implemented.

The design of the LL HSPA UL is totally based on 3GPP standards [1], [5]. It aims to implement the functionalities of all transport channel processing and spreading/modulation steps; moreover, the mobile radio channel behavior is modeled, with only Additive White Gaussian Noise (AWGN). HSPA UL is modeled through equivalent base-band representation of the signal in order to minimize the computational effort.

The LL HSPA UL simulator models the behavior of the mobile radio channel, physical layer up to coding, in order to evaluate the uplink radio performance for the E-DCH traffic channel. The focus was directed on E-DPDCHs, but the emulation of E-DPCCH is considered, i.e., the effects of E-DPCCH on E-DPDCH are taken into account, but E-DPCCH transmission/reception chain is not simulated. Compressed mode and pulse filtering aspects are not implemented as well as estimation of the radio channel is performed in an ideal way.

Several mathematical functions and specific classes and functionalities related to communications are primarily provided by the IT++ external library [10]. However, very specific functionalities, such as rate matching, segmentation, interleaving, etc., were implemented from scratch.
The simulator is composed by several entities, each one performing a particular activity in the entire link-level chain, such as channel coding, modulation, radio channel, among others. These entities are designed to act in a coordinated manner in LSDF. The corresponding reception functionalities are also designed. It is worthy to notice that only perfect estimations of the radio channel are considered by entities in the reception chain.

The modules' functionalities are independent of each other, but the interfaces between them are not, i.e., the data passed by one module to the adjacent one must respect the expected data type for that adjacent receiving module. The reception chain functionalities are performed by the same modules of the transmission chain, in accordance with the LSDF structure, i.e., each module executes the transmitting/receiving functionalities.

\section{LINK-TO-SYSTEM INTERFACE}

In order to reduce the whole computational effort of a complete network simulator, it is desirable to separate link and system-level functionalities into distinct simulator parts. Furthermore, we must define an efficient method for interfacing both parts. Generally, results of one set of link-level simulations - such as mean of BER, RBER and BLER can be abridged in look-up tables together with correspondent input parameters.

There are a number of L2S solutions, but we had to introduced another one that comprise the particularities of the used spreading codes. For the sake of simplicity, we have based on AVI approach [11]. Basically, the AVI method associates a single BLER value per average Signal-to-Noise Ratio (SNR). Note that channels whose fading velocities are distinct may be mistakenly seem to suffer the same rate of errors. However, while the studied channel is a simple AWGN, such approach is enough.

Fig. 4 shows a plain representation of AVI acting in a hypothetical curve of BLER $\times$ SNR for the $j^{t h}$-ETFCI. Before the SNR to BLER mapping takes place, a linear averaging is applied over the samples of instantaneous SNR taken at the time slot periods. This figure exemplifies the $10 \mathrm{~ms}$ TTI case.

It is worthy to notice that when Signal-to-Interference plus Noise Ratio (SINR) measurements are delivered to this L2S interface instead of the SNR measurements, the mapped BLER values correspond to the scenario in which the distribution of the interference is Gaussian. However, we know that a parcel of the other users' power will not introduce interference on the desired signal, since it is rejected by a correlation operation at the receiver. In this sense, while handling the SINR samples measured by the system-level part and SNR delivered to L2S interface, we model the interference reduction by means of an weighting factor, under the assumption of considering the interference parcel as Gaussian distribution. From now on, this weighting factor will be called Interference Reduction Factor $(I R F), \varrho$, whose influence is observed in:

$$
S N R_{L 2 S}=\frac{P_{D P C C H}^{\text {target }}}{\varrho \cdot I+N},
$$




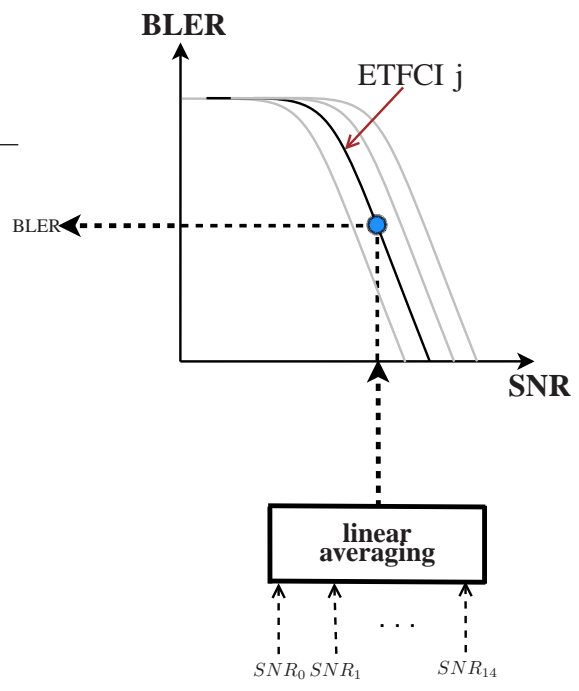

Fig. 4. Simplified single-step AVI adopted for HSPA UL simulations, $10 \mathrm{~ms}$ TTI, over channel with only AWGN.

where $P_{D P C C H}^{\text {target }}$ is the power of the DPCCH channel of the desired user averaged in one TTI, $I$ is the interference power and $N$ is the noise power.

Let $\mathbf{s}$ denote the desired user's Walsh-Hadamard code and $\mathbf{z}_{i}$ a complex pseudo-random code related to a certain interferer user $i$. We calculate the IRF values, $\varrho$, from:

$$
\varrho=\xi^{2},
$$

with $\xi=E_{i}\left|\Re\left\{\mathbf{s} \cdot \mathbf{z}_{i}\right\}\right|$, where $E_{i}\{\cdot\}$ denotes the expectation operator and $\Re\{\cdot\}$ denotes the real-part operator.

The cross-correlation values are the ones obtained from the operation performed by the correlator receiver in the presence of an interferer user.

We computed some values of IRF using a separate MATLAB ${ }^{\circledR}$ program, verifying a large number of situations. After, we sorted the values according to the spreading factor of the desired user. Likewise, the sorting of IRF according to the physical channel configuration can be deduced, as shown in Table I.

TABLE I

IRFS FOR HSPA UL.

\begin{tabular}{c|c}
\hline \hline Physical Channels Configuration & IRF \\
\hline \hline$N_{256}$ & $1.2412 \times 10^{-3}$ \\
\hline$N_{128}$ & $2.4825 \times 10^{-3}$ \\
\hline$N_{64}$ & $4.9254 \times 10^{-3}$ \\
\hline$N_{32}$ & $9.7373 \times 10^{-3}$ \\
\hline$N_{16}$ & $2.0598 \times 10^{-2}$ \\
\hline$N_{8}$ & $3.9916 \times 10^{-2}$ \\
\hline$N_{4}$ & $6.6461 \times 10^{-2}$ \\
\hline $2 \times N_{4}$ & $6.6461 \times 10^{-2}$ \\
\hline $2 \times N_{2}$ & $1.2500 \times 10^{-1}$ \\
\hline $2 \times N_{2}+2 \times N_{4}$ & $1.0345 \times 10^{-1}$ \\
\hline
\end{tabular}

Notice that the SNR of the desired user that is informed to the L2S interface is calculated considering the DPCCH power [see (2)]. Since there is a relation between the power of the
DPCCH and the power of the parallel E-DPDCHs within a UE, which is dependent on the $\beta_{e d, k}$ gain factors, we decided to simplify the L2S interface and perform the calculation of the $\beta_{e d, k}$ gain factors inside the LL HSPA UL simulator. Thus, the system-level simulator only needs to inform the L2S interface what are the SNR of the DPCCH channel, which is given by (2), and the E-TFCI of the user. Based on Fig. 3, the LL HSPA UL simulator calculates the combined power of the parallel E-DPDCHs using (4).

$$
P_{E-D C H}^{T T I}=P_{D P C C H}^{\text {target }} \cdot \sum_{k=1}^{K}\left(\frac{\beta_{e d, k}}{\beta_{c}}\right)^{2},
$$

where $P_{E-D C H}^{T T I}$ is the combined power of all E-DPDCHs of a given E-TFC during one TTI, $P_{D P C C H}^{\text {target }}$ is the power of the DPCCH channel of the desired user averaged in one TTI, and $\frac{\beta_{e d, k}}{\beta_{c}}$ is the power offset of the $k^{t h}$ E-DPDCH in relation to the DPCCH channel.

\section{Simulation Results}

The input parameters presented in Table II were used for a campaign of simulations of E-DCH link-level evaluation. Neither HS-DPCCH nor DPDCH are activated. For every tested E-TFCI, 15000 transport blocks were transmitted and received as a Monte Carlo simulation. The value considered for the puncturing limit $P L_{n o n-\max }$ was 0.84 , whereas the value of $P L_{\max }$ varied according to the $3 \mathrm{GPP}$ specification [1]. Moreover, we assume that the maximum spreading factor in the SET0 is $2 \times S F 4$.

As result, the simulation tool generated a BLER curve depicted in Fig. 5. Notice that the $E_{\text {chip }} / N_{0}$ is used instead of the SNR in the abscissa axis. The $E_{c h i p} / N_{0}$ metric in Fig. 5 is a channel quality measure seen by DPCCH. Notice that in case we want to obtain the channel quality measure seen by E-DPDCH, we must use (4).

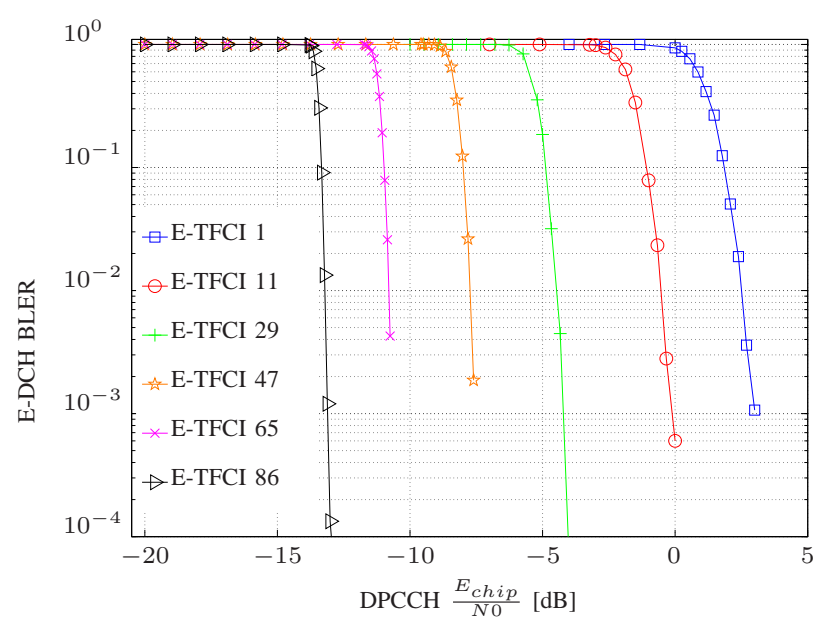

Fig. 5. BLER Curve.

Regarding the input parameters essential for the L2S interface, Table III gathers the set of configured E-TFCI indexes, their correspondent transport block size, their respective spreading factor, $\beta_{e d, j}$ gain factors [see (1)] and IRFs. These parameters are calculated offline using functionalities of the HARQ/RM and Spreading modules. 
TABLE II

LL HSPA UL SIMULATOR PARAMETERS.

\begin{tabular}{|c|c|}
\hline Parameter & Value \\
\hline \multicolumn{2}{|l|}{ General } \\
\hline Number of Iterations per $E_{\text {chip }} / N_{0}$ & 15000 \\
\hline Saving Period [iteration] & 100 \\
\hline HARQ offset Index $\left(\Delta_{\text {harq }}\right)$ & 0 \\
\hline Reference E-TFC Indexes & {$\left[\begin{array}{lllll}11 & 86 & 110 & 115\end{array}\right.$} \\
\hline $\begin{array}{l}\text { Reference gain factor for each } \\
\text { reference E-TFC }\left(\beta_{e d, r e f}\right)\end{array}$ & {$\left[\frac{9}{15} \frac{38}{15} \frac{53}{15} \frac{67}{15}\right]$} \\
\hline Power of HS-DPCCH and DPDCH [W] & 0 \\
\hline DPCCH gain factor $\left(\beta_{c}\right)$ & 1 \\
\hline E-DPCCH gain factor $\left(\beta_{e c}\right)$ & 1 \\
\hline CRC Code Type & WCDMA-24 \\
\hline TTI [ms] & 10 \\
\hline \multicolumn{2}{|l|}{ Code Block Segmentation } \\
\hline Minimum Block Size [bit] & 40 \\
\hline Maximum Block Size [bit] & 5114 \\
\hline \multicolumn{2}{|l|}{$\begin{array}{ll}\text { Turbo Coding } \\
\end{array}$} \\
\hline Generator polynomials (octal base) & $013015]$ \\
\hline Constraint Length & 4 \\
\hline Size of the turbo coding interleaving & 5114 \\
\hline Decoding algorithm & $\log -\max$ \\
\hline Decoding iterations & 8 \\
\hline \multicolumn{2}{|l|}{ HARQ/Rate Matching } \\
\hline $\begin{array}{l}\text { Set of } N_{e, \text { data }} \text { values allowed by } \\
\text { UTRAN and supported by UE }\end{array}$ & $N_{256}-2 \times N_{4}$ \\
\hline$P L_{n o n-\max }$ & 0.84 \\
\hline Redundancy Version Parameter & 0 \\
\hline \multicolumn{2}{|l|}{ Scrambling } \\
\hline Scrambling type & short \\
\hline Code length [chip] & 38400 \\
\hline Maximum Number of Codes & 16777215 \\
\hline
\end{tabular}

TABLE III

E-TFCI INFORMATION FOR L2S.

\begin{tabular}{r|r|r|r|c}
\hline \hline $\begin{array}{c}\text { E-TFCI } \\
\mathbf{j}\end{array}$ & \multicolumn{1}{|c|}{ TBS } & SF & $\beta_{\text {ed, }, \text { harq }}$ & IRF \\
\hline \hline 1 & 120 & SF64 & 0.490 & $4.9254 \times 10^{-3}$ \\
\hline 11 & 180 & SF32 & 0.600 & $9.7373 \times 10^{-3}$ \\
\hline 29 & 374 & SF16 & 0.865 & $2.0598 \times 10^{-2}$ \\
\hline 47 & 776 & SF8 & 1.246 & $3.9916 \times 10^{-2}$ \\
\hline 65 & 1613 & SF4 & 1.796 & $6.6461 \times 10^{-2}$ \\
\hline 86 & 3784 & $2 \times$ SF4 & 2.533 & $6.6461 \times 10^{-2}$ \\
\hline 110 & 10028 & $2 \times$ SF4 & 3.533 & $6.6461 \times 10^{-2}$ \\
\hline 115 & 12286 & $2 \times$ SF4 & 4.467 & $6.6461 \times 10^{-2}$ \\
\hline \hline
\end{tabular}

\section{Conclusions And Perspectives}

The single-user LL HSPA UL simulator is characterized by the modularity and reusability. The programming framework is completely implemented in $\mathrm{C}++$ language based on OOP concepts. One remarkable characteristic of the simulator is the flexibility to take advantage of external mathematical and telecommunications libraries, since only the common interface among data blocks and building modules should be respected. In this way, IT++ external libraries could be used effectively and easily.

The LL HSPA UL simulation tool was developed in accordance to the 3GPP standards. Data blocks are transmitted throughout the complete coding/multiplexing/spreading/modulation chain, under an AWGN radio channel. The reverse process is performed through the respective reception chain and errors are computed. As a Monte Carlo simulation, the link is evaluated for a large number of transport blocks. At the end of a campaign of simulations, BLER and BER curves are available as well as an LuT that will be used by a system level HSPA UL simulator.

Regarding the L2S interface, we suggested the use of a modified AVI, since only AWGN radio channel was investigated. We introduced the IRF concept in order to model the interference rejection at the correlator receiver. The IRF values are computed separately generating a special LuT that relates the E-TFCI and IRF. A simple L2S interface was designed assuming as input parameters the DPCCH channel quality and the desired user's E-TFCI. Furthermore, there is the perspective to modify other L2S interface solutions through the inclusion of the IRF concept.

The built HSPA UL simulation tool allows a number of possible applications. Sophisticated channel receivers/equalizers and space diversity are examples of further studies and features that can be performed. Moreover, the inclusion of the Incremental Redundancy functionality besides an efficient L2S interface can be performed, yielding some additional investigation topics such as retransmission and interference (e.g., multipath and multiuser interferences) modeling.

\section{REFERENCES}

[1] 3GPP. Multiplexing and channel coding (FDD). Technical Specification TS 25.212 V6.7.0 - Release 6, $3^{\text {rd }}$ Generation Partnership Project, Sophia Antipolis, France, December 2005.

[2] Stefan Parkvall, Janne Peisa, Johan Torsner, Mats Sågfors, and Peter Malm. WCDMA Enhanced Uplink - Principles and Basic Operation. In IEEE 61st Vehicular Technology Conference, 2005. VTC 2005-Spring, volume 3, pages 1411-1415, May 2005.

[3] Eva Englund, Y.-P Eric Wang, Christer Edholm, Jung-Fu Cheng, Carmela Cozzo, Maria Edvardsson, and Ke Wang Helmersson. High-Data-Rate Availability in WCDMA Enhanced Uplink Systems. In IEEE 62nd Vehicular Technology Conference, 2005. VTC 2005-Fall, volume 4, pages 2477-2481, September 2005.

[4] Harri Holma and Antti Toskala, editors. HSDPA/HSUPA for UMTS: High Speed Radio Access for Mobile Communications. John Wiley \& Sons, Ltd, May 2006.

[5] 3GPP. Spreading and modulation (FDD). Technical Specification TS 25.213 V7.0.0 - Release 7, $3^{\text {rd }}$ Generation Partnership Project, Sophia Antipolis, France, March 2006.

[6] Stefan Parkvall. WCDMA Enhanced Uplink - Technical Description (Release 6). Technical Report EAB-04:013098 Uen, ERICSSON, December 2004.

[7] 3GPP. Feasibility Study for Enhanced Uplink for UTRA FDD. Technical report, $3^{\text {rd }}$ Generation Partnership Project, 3GPP TR 25.896 version 6.0.0 Release 6, April 2004

[8] 3GPP. Physical Layer Procedures (FDD). Technical Report TS 25.214 v7.1.0 - Release 7, $3^{\text {rd }}$ Generation Partnership Project, Sophia Antipolis, France, June 2006.

[9] C. H. M. de Lima, E. M. G. Stancanelli, E. B. Rodrigues, J. M. S. Maciel, and F. R. P. Cavalcanti. A Software Development Framework based on C++ OOP language for link-level simulation tools. ITS2006 International Telecommunications Symposium, September 2006.

[10] Free Software Foundation. IT++ - Scientific Library, 2001. http://itpp.sourceforge.net, accessed on March 07, 2006.

[11] A. Furuskar. Radio Resource Sharing and Bearer Service Allocation for Multi-Bearer Service, Multi-Access wireless networks - methods to improve capacity. $\mathrm{PhD}$ thesis, Royal Institute of Technology - KTH, 2003. 